\title{
Path Analysis of the Impact of COVID-19-related Stress Response on Phobia and Anxiety Experienced by College Students
}

\author{
Fei Wang*, Ping-Ting Pei, Xin Wang, Jing Wang, Ya-Xin Qian and Heng-Yu Hu \\ School of Philosophy, Anhui University, Hefei, Anhui, China \\ ${ }^{\star}$ Corresponding author: Fei Wang, School of Philosophy, Anhui University, Hefei, Anhui, China; Tel: 13083163161; Email: 3173363852@qq.com
}

Received: August 08, 2021; Accepted: August 14, 2021; Published: October 22, 2021

\begin{abstract}
Objective: This study aimed to explore the effects of the different dimensions of psychological stress on the horror and anxiety experienced by college students during the COVID-19 pandemic.
\end{abstract}

Methods: The convenient sampling method was employed to select 169 respondents for the questionnaire survey.

Results: The correlation coefficients between the variables were significant, and the path analysis model registered satisfactory fitness.

Conclusion: Panic and Defensiveness resulting from psychological stress can directly affect anxiety, and defensiveness can also indirectly affect anxiety through horror. Conversely, cognition can only function in a completely mediating role in the effects of psychological stress on anxiety through horror.

Keywords: Psychological stress, COVID-19 pandemic, Panic, Defensiveness, Anxiety

\section{Introduction}

The severity of the COVID-19 outbreak that first occurred in December 2019 transcended expectations and attracted much attention from all sectors of society. The World Health Organization identified it as a "public health emergency of international concern." Relevant studies have demonstrated that major infectious disease epidemics are likely to seriously damage and influence human physical and mental health [1]. The COVID-19 pandemic is associated with stress in the general public from two studies from different regions [2,3]. A study of pregnant women found that Chinese pregnant women were affected by moderate to severe stress during the COVID-19 pandemic period [4].

Compared with ordinary people, in order to protect the health of college students, the government has implemented the policy of "classes suspended but learning continues". As the longest isolated group in the country, college students' mental health will inevitably be affected. For example, the COVID-19 pandemic is associated with severe anxiety symptoms [5]. Some scholars have suggested that the spread of COVID-19 and the resulting obstructions could cause a negative impact on the mental health of adolescents [6]. The occurrence of public emergencies can generate stress responses in the general public. Some researchers divide this stress response vis-à-vis the epidemic situation into three dimensions: panic is the most important component, followed by the defense response; finally, cognition can discharge a significant role in the regulation and inhibition of the stress response [7].
The extant literature suggests that public exposure to COVID-19 could lead to serious mental health problems, including mood disorders, anxiety disorders, and panic attacks [8]. Psychological stress manifests in different ways in emotional cognition and physiological functions. Previously conducted studies have found that emotional problems become prominent during public emergencies occur [9]. Irrational emotions could lead to anxiety. Individual premonitions of unfavorable situations result in mental anxieties that become visible as unhappiness, inner tension, irritability, and so on [10]. Studies have found that individuals could exhibit a certain intensity of phobia symptoms during the COVID-19 pandemic [11], and that some people could show strong fear toward triggers such as open spaces, public places, travel, other people, and vehicles which could further cause them to experience anxiety.

Scant studies currently exist on the mechanism of the stress response affecting anxiety. However, some researchers have found that the Symptom Checklist-90 (SCL-90) phobia factor demonstrates a significant positive correlation with stress intensity in four kinds of life events [12]. Studies have also evidenced that cognitive reappraisal can regulate individual phobia symptoms to a certain extent [13]. The SCL-90 phobia factor is also found to correlate significantly with various factors of defense style [14]. Therefore, the present study explores the differences observed in psychological stress in its different dimensions with respect to phobia and anxiety levels. It analyzes the path of SCL-90 phobia and anxiety in different response modes to COVID-19. In so doing, it offers an investigative basis for the application of relevant measures to alleviate the anxiety of the people during the ongoing COVID-19 crisis. 


\section{Methods}

\section{Research Objects}

Convenience sampling was employed to distribute a total of 169 questionnaires to college students from high incidence (Hubei Province) and low incidence (Anhui Province) areas. The study was conducted from May 17, 2020 to May 28, 2020. In this study, only Chinese non pregnant local college students who lived in Wuhan, Hubei province or Hefei, Anhui Province from December 2019 to may 2020 (i.e. during the pandemic) and could give informed consent were recruited. All participants provided informed consent before participating in the study. All participants in this study had no history of mental illness. The internal part of the questionnaire is set up with lie detection questions, and the exclusion criteria are wrong lie detection questions and inconsistent logic. This research protocol has been approved by the ethics committee of Anhui University.

\section{Measurement Instruments}

\section{Psychological Stress Response Questionnaire}

Tong Huijie developed the psychological stress response questionnaire [7], which comprised a total of 17 items measuring three dimensions. Firstly, there are 5 entries for COVID-19's cognitive assessment: "I believe mankind will conquer the epidemic." Secondly, there are six items in panic about the epidemic situation, such as "the worry about the epidemic situation makes me feel cold sweat or shiver sometimes". Finally, there are six items in the defensive psychological and behavioral response to the epidemic situation, such as "to prevent the epidemic situation, I will wear masks in public places". The questionnaire adopts 4-point scoring, three of which are reverse scoring. The higher the score is, the more serious the individual's stress response is. In this study, the internal consistency coefficients of panic, defense and cognition were 0.72 , 0.74 and 0.71 respectively.

\section{Symptom Checklist-90}

DeRogatis et al. compiled the SCL-90 in 1973. It was translated into China and widely used in the field of mental health [15]. The scale includes 90 items and 9 subscales, namely somatization, obsessivecompulsive symptoms, interpersonal sensitivity, depression, anxiety, hostility, phobia, paranoia and psychoticism. In this study, 7 items of phobia subscale are selected, which is basically consistent with the traditional state of terror or square terror. The scale adopts a five point score of " $0-5$ ", and the total score of the sub scale is used to measure the individual's degree of terror. The higher the total score is, the more serious the degree of terror is. In this study, the internal consistency coefficient of the scale was 0.92 .

\section{Self-rating Anxiety Scale (SAS)}

SAS encompasses 20 items and was compiled by Chinese American professor Zung in 1971. SAS is used to assess the anxiety status of subjects by probing their perceptions about their experiences over the past week. It is widely applicable and germane to adults evincing anxiety symptoms [16]. The Cronbach alpha coefficient of this scale was 0.84 for the present study.

\section{Statistical Analysis}

SPSS22.0 and Amos22.0 were employed for the data analysis. Descriptive statistics were utilized to demonstrate the socialdemographic characteristics of samples. Pearson correlation analysis was deployed to analyze the correlations between the variables. According to the hypothesis model, path analysis tests the relational model, and multiple fitting indices were utilized to evaluate the adequacy of the model. Absolute fit indices included chi-square statistic $(p>0.05)$, the Root Mean Square Error of Approximation (RMSEA < 0.08), Goodness-of-Fit Index (GFI > 0.9), and Adjusted Goodness-of-Fit Index (AGFI $>0.9$ ). Incremental fit indices contain Normed Fit Index (NFI > 0.9), Relative Fit Index (RFI > 0.9), Incremental Fit Index (IFI $>0.9$ ), Tacker-Lewis Index (TLI $>0.9$ ) and Comparative Fit Index (CFI $>0.9)$. Parsimonious fit indices encompassed normed chi-square $(\chi 2 / d f<2)$, It is generally believed that path analysis can be carried out when the sample size is more than 100 [17].

\section{Results}

\section{Sample Description}

Among 169 college students who were invited to complete the questionnaire, 119 were included in the final analysis, with a completion rate of $70.4 \%$ (Table 1) and the inclusion process is shown in Figure 1. Those who declined the invitation explained that they didn't have much time to participate. The mean age of the participants was 20.1 \pm 2.3 years. Most of the subjects were female. Among all participants, liberal arts major accounted for the largest proportion (47.9\%), as the same time, the majority of college students (77.3\%) live between 1000 and 2000 yuan per month.

\section{Correlations among Study Variables}

Pearson correlation coefficient was utilized for the analysis of the correlations between the study variables. Table 2 elucidates

Table1: Social-demographic characteristics of participants.

\begin{tabular}{|l|c|c|}
\hline Variables & Level & $\mathbf{n}(\%) /$ Mean \pm SD \\
\hline Ages & & $20.1 \pm 2.3$ \\
\hline Gender & Male & $38(32.0 \%)$ \\
\hline Region & Female & $81(68.1 \%)$ \\
\hline & High incidence area & $15(13.0 \%)$ \\
\hline Major type & Low incidence area & $104(87.4 \%)$ \\
\hline & science & $34(28.6 \%)$ \\
\hline & Liberal arts & $57(47.9 \%)$ \\
\hline Monthly living expense & engineering & $28(23.5 \%)$ \\
\hline & $<1000$ yuan & $12(10.1 \%)$ \\
\hline & $1000 \sim 1500$ yuan & $42(35.2 \%)$ \\
\hline history of mental health disease & $1500 \sim 2000$ yuan & $50(42.0 \%)$ \\
\hline & $>2000$ yuan & $15(12.7 \%)$ \\
\hline
\end{tabular}




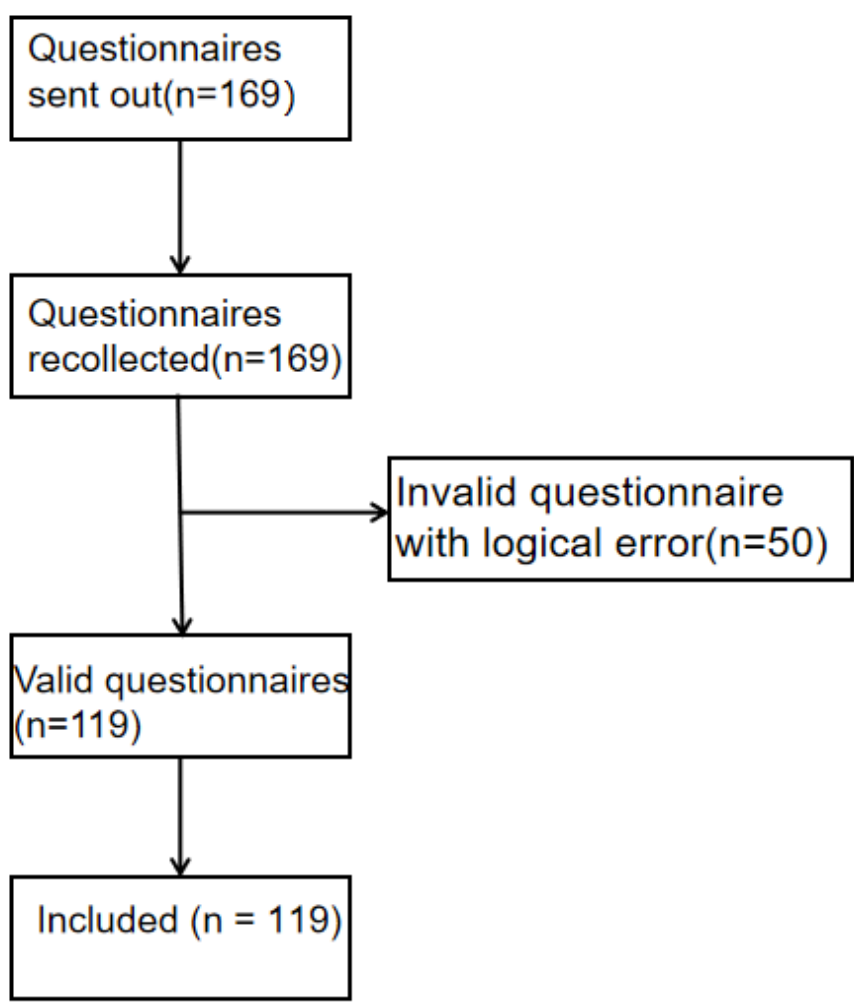

Figure 1: Flowchart of the participant inclusion process.

Table2: Correlation coefficient among study variables.

\begin{tabular}{|l|c|c|c|c|c|}
\hline & Panic & Defense & Cognition & Phobia & Anxiety \\
\hline Panic & 1 & & & & \\
\hline Defense & $0.44^{* *}$ & 1 & & & \\
\hline Cognition & $-0.20^{*}$ & -0.04 & 1 & & \\
\hline Phobia & $0.28^{* *}$ & $0.35^{* *}$ & $-0.21^{*}$ & 1 & \\
\hline Anxiety & $0.39^{* *}$ & 0.05 & $-0.23^{*}$ & $0.44^{* *}$ & 1 \\
\hline
\end{tabular}

Note: ${ }^{*} \mathrm{P}<0.05,{ }^{* *} \mathrm{P}<0.01$. that a significant positive correlation was found between panic, defensiveness, phobia, and anxiety, and a significant negative correlation was discerned between cognition and fear and between phobia and anxiety.

\section{Effects of Stress Response Patterns on Anxiety}

Panic, defense and cognition are the three dimensions of the stress response model. Considering that the stress response may affect individual anxiety through the mediating of terror, the model was established after several adjustments. Fit indices revealed that the path model obtained a satisfactory fit to the data. $\chi 2=2.65(p=0.27)$, $\chi 2 / d f=1.33$. RMSEA $=0.05, \mathrm{GFI}=0.99, \mathrm{AGFI}=0.93$. The values of four incremental fit indices values were computed between 0.96 and 0.99 . The results revealed that panic $(\beta=0.40, p<0.001)$ and defensiveness $(\beta=-0.27, p<0.01)$ had direct effects on anxiety. Defensiveness could also mediate anxiety through SCL-90 phobia $(\beta=0.15, p<0.01)$, by contrast, the direct and indirect effects of defense reaction are not consistent. The direct effect has a negative impact on anxiety, while the indirect effect of terror has a positive impact on anxiety. Besides, cognition $(\beta=-0.08, p<0.05)$ could only affect anxiety through the complete mediating effect of SCL-90. Figure 2 demonstrates the output path graph: the path coefficients inscribed on each path were calculated. Table 3 displays the total, direct, and indirect effects of the variables in this model.

Table 3: Total, direct, and indirect effect of variables in this Model.

\begin{tabular}{|l|c|c|c|}
\hline Independent/dependent variable & Direct effect & Indirect effect & Total effect \\
\hline Panic/anxiety & 0.40 & & 0.40 \\
\hline Defense/anxiety & -0.27 & 0.45 & 0.18 \\
\hline Defense/SCL-90 phobia & 0.34 & & 0.34 \\
\hline Cognition/anxiety & & -0.26 & -0.26 \\
\hline Cognition/SCL-90 phobia & -0.19 & & -0.19 \\
\hline SCL-90 phobia/anxiety & 0.43 & & 0.43 \\
\hline
\end{tabular}

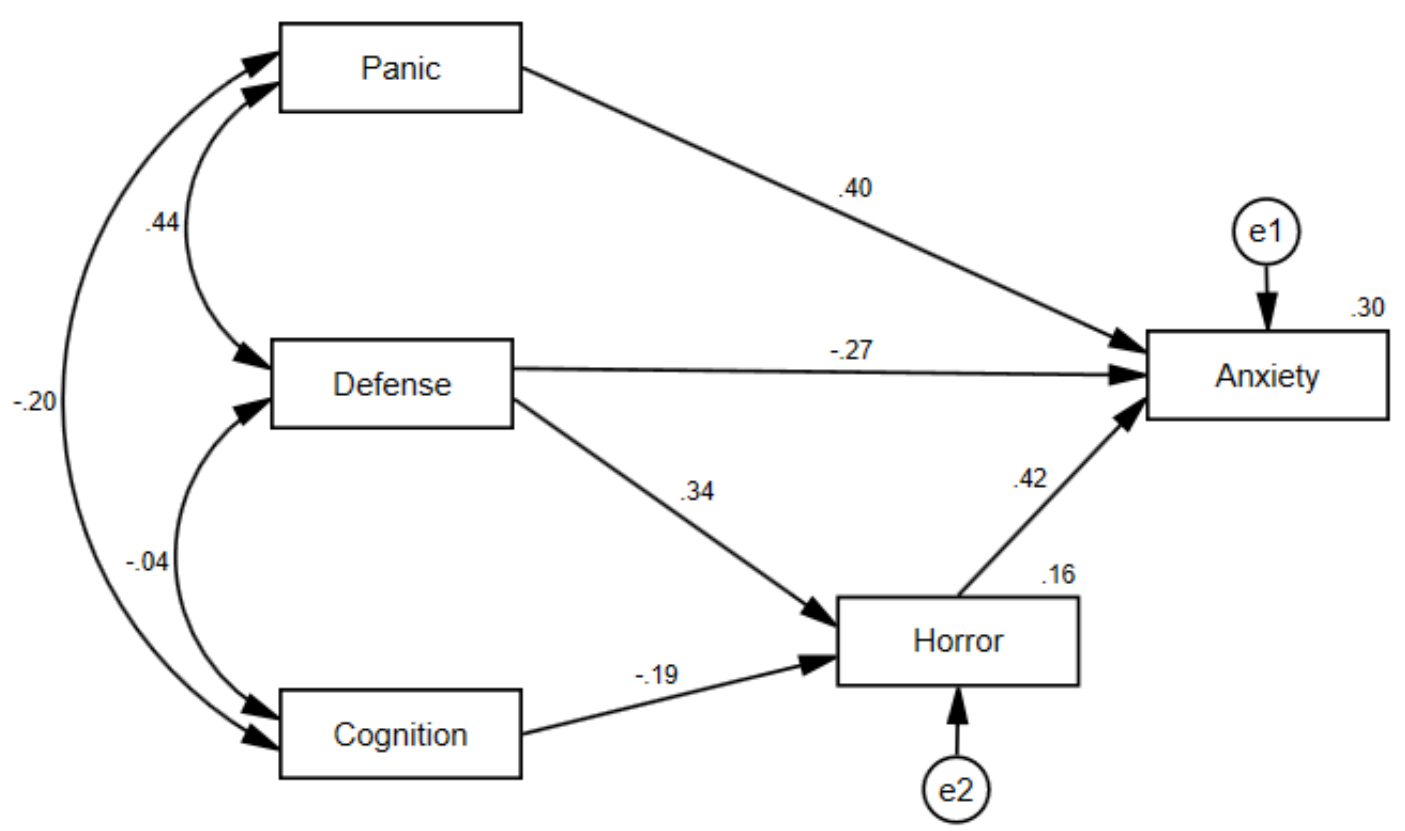

Figure 2: Path analysis of psychological stress and anxiety. 


\section{Discussion}

This study mainly probes into that the defensive psychology and behavior in the epidemic psychological stress will have different impacts on anxiety among the group of college students. Defensive psychology and behavior have a direct negative impact on anxiety, which means the higher the defense, the lower the anxiety of the individual performance. But yet in the indirect effect of defense on anxiety, it is showed that defensive psychology and behavior can give rise to enhancement of the individual's phobia psychology, which will result in further deepen the individual's anxiety. Moreover, the higher the defensive psychology and behavior in terms of total effect is, the higher the individual anxiety is. Preceding studies have proposed that there is a significant negative correlation between the protective compliance behavior and anxiety of ordinary people who quarantined at home at the early stage of the epidemic [18], the higher the defensive behavior, the lower the anxiety state. It's worth noting that the research object of this study is college students. Contrasred with other groups, college students are characterized by quick acceptance of new information and strong learning ability. Therefore, in the face of various prevention measures of COVID-19 from social media, college students are inclined to take actions instantaneously and emphasize more on comprehensive implementation. James Langer Emotion Theory believes that the changes of body will directly affect the individual's emotions, the external behavior of the individual will have an impact on the individual's emotions [19]. Thus compared with other groups, college students' defensive psychology and behavior are higher, and their anxiety and phobia of the epidemic are also higher. In addition, time is also an important factor in the relationship between defense and anxiety. This study was carried out in the early and middle stages of the epidemic. Previous studies have demonstrated that in the later period of the epidemic, individual phobia decreased significantly [20]. Due to in the later period of the epidemic in China, the epidemic has taken a turn for the better, and the phobia of college students also gradually anesised. Their defensive behavior was not as strong as that during the epidemic, and they were aware of the protective effect of these behaviors on COVID-19, showing that the defensive behavior of college students negatively predicted anxiety.

This study also revealed that in college students, cognition in psychological stress can merely act negatively on anxiety through the complete mediator of phobia. There are plenty of previous studies which have found that incorrect epidemic cognition is significantly correlated with the occurrence of anxiety [21]. In the meantime with high cognition of COVID-19, college students tend to change of health behavior in order to reduce the anxiety [22]. Reasonable emotion theory holds that people's cognition, emotion and behavior are causeand-effect related, and suggests that emotional state and behavior performance are result from thought, belief and way of thinking, that is to say individual unreasonable belief is the cause of individual phobia and anxiety. Different from the direct effect of cognition and anxiety found in the non-acute anxiety study, the main reason for anxiety in the acute stress raised from the epidemic is no longer the individual's unreasonable belief. Therefore the cognition in the stress response model of the epidemic does not have an direct influence on anxiety.
However, some studies have found that during the epidemic period, people's acquisition of misinformation and misinterpretation may result in phobia [23]. But the college student's ability of information acquisition is much better than that of the general public. Therefore, among college students, the negative effect of cognition on anxiety is manifested as that individuals with incorrect cognition will show higher phobia psychology, and both phobia and anxiety are belong to anxiety disorders [24]. Such phobia of the epidemic will provoke the individual to be more anxious.

With the exception of the above conclusions, this study found that panic in psychological stress can significantly positively predict individual anxiety among college students, which is consistent with the conclusion of previous studies. Previous studies have found that people with high panic have higher anxiety in the acute stress response [7], and it is anxiety that is one of the most prominent psychological characteristics of college students in the panic period of psychological stress response during the epidemic [5]. This is probably owe to phobia and anxiety in COVID-19 existing in parallel [25], and simultaneously the parallel existence of phobia and anxiety can further contribute to each other, leading to greater phobia and anxiety.

Through the method of path analysis, this study found different dimensions of the mechanism of action of individual anxiety in groups of college student's psychological stress, revealing the roles that phobia psychology plays in defensive psychology and behavior of psychological stress and that cognitive dimension plays. All above this are paving the way and providing a train of thought for college students to overcome anxiety of acute psychological stress.

Nonetheless this study also has several limitations. On one hand, this study is a cross-sectional study so that subsequent studies can further explore the effect of time on the mechanism of psychological stress and anxiety which has not be talked over in the study. On the other hand, the quantity of subjects in this study is relatively insufficient and hence later studies can further enrich the amount of subjects.

\section{Conclusion}

In college students, the defensive psychology and behavior in epidemic psychological stress will have different effects on anxiety. The direct effect is that the higher the defensive psychology and behavior, the lower the anxiety. The indirect effect is that the higher the defensive psychology and behavior, the higher the terror, and the higher the anxiety. In the early and middle stages of the epidemic, the direction of total effect and indirect effect is the same. Besides, in college students, Cognition in situations of psychological stress further influences anxiety by being a complete mediator of phobia. Finally, in college students, panic in psychological stress can significantly increase individual anxiety.

\section{References}

1. Zhu X, Hu Y, Wang TT, Zhong J, Yan YP, et al. (2020) Psychological symptoms of the public during the COVID-19 epidemic. Chinese Journal of Mental Health 34: 703-709.

2. Cao Y, Ma ZF, Zhang Y, Zhang Y (2020) Evaluation of lifestyle, attitude and stressful impact amid COVID-19 among adults in Shanghai, China. International Journal of Environmental Health Research 1-10. [crossref] 
Fei Wang (2021) Path Analysis of the Impact of COVID-19-related Stress Response on Phobia and Anxiety Experienced by College Students

3. Ma ZF, Zhang Y, Luo X, Li X, Li Y, et al. (2020) Increased stressful impact among general population in mainland China amid the COVID-19 pandemic: a nationwide cross-sectional study conducted after Wuhan city's travel ban was lifted. International Journal of Social Psychiatry 66: 770-779. [crossref]

4. Zhang Y, Ma ZF (2020) Psychological responses and lifestyle changes among pregnant women with respect to the early stages of COVID-19 pandemic. International Journal of Social Psychiatry 67: 344-350. [crossref]

5. Liu SM, Yuan Y, Luo B (2020) Influence of COVID-19 on Depression and Anxiety of College Students and Analysis of Related Factors. Journal of Clinical Research 37: 819-823.

6. Sélim Benjamin Guessoum, Jonathan Lachal, Rahmeth Radjack, Emilie Carretier, Sevan Minassian, et al. (2020) Adolescent psychiatric disorders during the COVID-19 pandemic and lockdown - ScienceDirect. Psychiatry Research 291: 113264.

7. Tong HJ (2004) Model of SARS stress and it's character. Journal of Psychology 01: 103-109.

8. Hossain MM, Tasnim S, Sultana A, Faizah F, Mazumder H, et al. (2020) Epidemiology of mental health problems in COVID-19: a review. F 1000 Research 9: 636. [crossref]

9. Wang YN, Luo YJ (2003) Specialty of mood disorders and treatment during emergent events of public health. Advances in Psychological Science 4: 387-392.

10. Zhang K (2003) Healthy mind conquers SARS: psychological response to SARS. Science Press.

11. Zhang CY, Peng XB, Zhang L, et al. (2020) Novel coronavirus pneumonia during the first aid nurses in Wuhan: mental health status and coping styles. Journal of Qilu Nursing 26: 7-10.

12. Jing SJ (2000) A study on the relationship between stress intensity of daily life events and mental health of junior middle school students. Youth research 5: 22-26.

13. Cheng L, He YY, OuYang HL, et al. (2011) Regulation of terror: cognitive reappraisal is better than expression inhibition. Psychological Science 4: 925-930.
14. Zhang BF, Qian WZ, Yu W, et al. (2006) The study on the mental health level of students in middle school and the related factors. Nervous Diseases and Mental Health 6: 178-179.

15. Wang XD, Wang XL, Ma H (1999) Rating Scales for Mental Health, revised version.

16. Li WH, Huang Y, Wang CL, et al. (2020) shelter hospital anxiety state of New Coronavirus pneumonia patients. China Journal of health psychology 28: 1191-1194.

17. Wu ML (2009) Structural equation modeling-Operation and application of AMOS.

18. Li J, Wang SH, Yu RH, et al. (2020) Compliance of novel coronavirus pneumonia during home quarantine and its impact on anxiety level. Chinese Journal of infection control 19: 26-32.

19. Peng DL (2001) General Psychology (Revised Edition). Beijing Normal University Press.

20. Su BY, Ye YX, Zhang W, et al. (2020) Time Course Characteristics of the Public's Psychological Symptoms during Different Stages of the Outbreak of COVID-19. Journal of South China Normal University (SOCIAL SCIENCE EDITION) 03: 79-94.

21. Liu ZR, Zhang XD, LÜ ZH, Liang JE, Deng Y, et al. (2020) Mental health status and its influencing factors among general population and medical personnel in Guangdong Province during COVID-19 pandemic. J South Med Univ 40: 1530-1538. [crossref]

22. Chang JH, Yuan YX, Wang D (2020) Mental health status and its influencing factors among college students during the epidemic of COVID-19. J South Med Univ 40: 171-176. [crossref]

23. Zhang J, Liu ZX, Liu YY, et al. (2020) Status and emotional impact of popular information acquisition under a novel coronavirus pneumonia outbreak. Journal of Jinan University 41: 58-64.

24. Wang X, Zhang JS (2010) Application of event-related potential in neuropsychological study of anxiety disorders in children. Shanghai Psychiatric Medicine 22: 50-52.

25. Kong ZM (2020) Media panic caused by epidemic events and modern governance. Yunnan Social Sciences 235: 178-183. 